Revista Calidad en la Educación Superior

Programa de Autoevaluación Académica

Universidad Estatal a Distancia

ISSN 1659-4703

Costa Rica

revistacalidad@uned.ac.cr

PERSPECTIVAS DE LA EVALUACIÓN UNIVERSITARIA: UN RETO PARA LA EDUCACIÓN SUPERIOR Y LOS ORGANISMOS DE ACREDITACIÓN EN LA SOCIEDAD DEL CONOCIMIENTO

PROSPECTS OF UNIVERSITY ASSESSMENT: A CHALLENGE FOR HIGHER EDUCATION AND ACCREDITATION AGENCIES IN THE SOCIETE OF KNOWLEDGE

Dr. Guillermo León Londoño Restrepo

Miembro del Consejo de Acreditación del CNA

Ex Vicerrector de Docencia la Universidad de Antioquia

Volumen 1, Número 1

Mayo 2010

pp. 1 - 12

Recibido: abril, 2010

Aprobado: mayo, 2010 


\title{
PERSPECTIVAS DE LA EVALUACIÓN UNIVERSITARIA: UN RETO PARA LA EDUCACIÓN SUPERIOR Y LOS ORGANISMOS DE ACREDITACIÓN EN LA SOCIEDAD DEL CONOCIMIENTO
}

\section{Resumen}

El artículo ofrece una perspectiva de la evaluación universitaria en donde la acreditación y la evaluación son dos dispositivos estratégicos que permiten orientar los desarrollos de la institución y de los programas, como instrumentos que la institución acoge y los utiliza según sus intereses y condiciones específicas.

Sostiene que la educación no es responsabilidad exclusiva del Estado, de la sociedad o de la institución educativa, sino responsabilidad de todos, de manera compartida.

Entendidos así los ejercicios de auto evaluación y evaluación externa y las relaciones que entre ellas se establecen se hace claro el principio de que "la evaluación es el ejercicio de la academia para examinar la academia" y que al Estado y a los entes externos sólo les es dado armarse de los criterios y mecanismos que aquella les proporciona.

Establece los márgenes de accionar de la universidad y de los entes acreditadores, como la responsabilidad compartida y los límites en el accionar.

Palabras claves: autoevaluación, acreditación, evaluación externa, educación superior, calidad.

\begin{abstract}
The article offers an overview of the university evaluation where the accreditation and evaluation are two strategic devices capable of guiding the development of the institution and programs, including instruments that the institution welcomes and uses them according to their interests and specific conditions.
\end{abstract}

It argues that education is not the sole responsibility of the State, society or school, but everyone's responsibility, a shared basis.

Well understood self-assessment exercises and external evaluation and the relationship between them becomes clear down the principle that "the assessment is the practice of the academy to examine the academy" and that the state and external entities is their only muster given the criteria 
and mechanisms that provide them.

Set the margins of the triggering of the university and accrediting bodies, such as shared responsibility and the limits on power.

Keywords: self-assessment, accreditation, external evaluation, higher education quality.

\section{¿Qué decimos cuando hacemos referencia a la evaluación universitaria?}

Se hace referencia a un ejercicio académico complejo que se propone una revisión crítica integral de la institución tomada como un todo, o de alguna de sus funciones sustantivas, de sus dependencias académicas o administrativas o de sus programas de formación y que se propone producir información completa, válida y confiable que permita orientar decisiones adecuadas para el mejoramiento y desarrollo del objeto o de los objetos evaluados.

\section{Ejercicio académico}

Se entiende la evaluación como un ejercicio de la academia para examinar la academia, como una herramienta reconocida, adoptada y fomentada por el Estado y otros organismos en el marco de sus políticas de apoyo a las instituciones y de fomento de su calidad, para la preservación de la institución universitaria el estado se arma de los criterios de saber que le proporciona la propia universidad. La evaluación se toma ejercicio académico, no burocrático, diseñado y desarrollado por académicos para el mantenimiento y reproducción de la propia institución.

\section{Complejo}

Por el entendimiento de la institución como un ente histórico, determinado por los desarrollos científicos, sociales, económicos y culturales, por los múltiples actores que involucra, por los componentes técnicos y políticos que lo cruzan, por la diversidad y complejidad de las decisiones que orienta, por la gestión administrativa que requiere y por la variedad de recursos que demanda.

\section{Revisión crítica}

Determinada por la comprensión de la institución, sus funciones, dependencias o programas, como constituidos por la interrelación de múltiples factores, de la cual se espera una aprehensión sintético analítica completa y no la simple descripción o conocimiento parcial.

Que se propone producir información completa, válida y confiable. Significa que es un proceso racional, metodológico y técnicamente desarrollado; que se apoya en fuentes, técnicas de recolección 
de información y métodos de análisis validos.

Que permite orientar e informar decisiones adecuadas para el mejoramiento y desarrollo del objeto evaluado. Implica que la evaluación se desarrolla en un marco institucional que tiene como uno de sus fundamentos la voluntad política y administrativa para cambiar y remover las debilidades que se hallen y consolidar las fortalezas que se identifiquen, implica que la evaluación no es un fin en sí, ella es un dispositivo institucional para su conocimiento y mejoramiento y que si no existe la voluntad y no se proveen las capacidades para el mejoramiento ese ejercicio no dejará de ser retórica institucional y en vez de lograr orientar avances corre el riesgo grande de marcar retrocesos de la institución, de sus funciones, dependencias y programas.

Si la evaluación no se desarrolla con el propósito del mejoramiento no tiene sentido. Se pierden esfuerzos, recursos, tiempo, se desgasta este dispositivo como algo útil para la gestión institucional y sobre todo se menoscaba la confianza que debe existir entre los actores institucionales y las directivas centrales de la institución y las de las unidades académicas y entre las instancias y órganos de dirección de la institución.

\section{"Evaluación universitaria"}

Señala a la Universidad como foco de estudio. Ella debe ser entendida por su esencia., como una institución se saber, como una institución que se ocupa de los problemas fundamentales de la sociedad, en la universidad buscamos la sabiduría a ella se le confía la generación del saber que la sociedad considera especializado y, en consecuencia., también la formación de los especialistas. El ejercicio soberano de ese saber que se le reconoce a la universidad constituye su autonomía, fuero reivindicado para mantenerse a salvo de la interferencia de poderes externos a ella.

La cualidad primera de la institución universitaria, de la universidad, la referencia a la producción del conocimiento, a la búsqueda racional de la verdad como su interés primero, la hace manifiesta por la investigación, por el ejercicio de la crítica, por el ejercicio de las libertades de pensamiento, de cátedra y de aprendizaje: ello no excluye su carácter de institución de servicio a la sociedad en tanto el conocimiento tiene una función social y se realiza en sus aplicaciones prácticas a la solución de los problemas sociales y económicos.

La Universidad presta la educación como un servicio público, ésta es un bien público e individual, es un servicio público cultural, a través del cual se procura el acceso al conocimiento, a la ciencia, a la 
técnica y a los demás bienes y valores de la cultura. Al evaluar la Universidad no puede pues excluirse el vínculo de la universidad con las urgencias y demandas de la sociedad; al dotarla de un ideal de servicio a la sociedad debe reconocerse y respetarse la legitimidad de su pensamiento.

\section{¿Porqué evaluar la universidad, cómo se entiende la educación hoy y qué demandas se le hacen?}

En las últimas décadas la sociedad ha venido experimentado cambios acelerados y ha hecho que los sistemas educativos en su evolución hayan experimentado transformaciones significativas, la educación superior, con ella la institución universitaria, ha sido objeto de las más diversas demandas por parte de los Estados y gobiernos, de los sectores productivos de bienes y servicios, y por la sociedad en su conjunto.

La sociedad actual se ha caracterizado por un fuerte dinamismo expresado, entre otros fenómenos, por:

El crecimiento demográfico.

Continúo ascenso de los estratos inferiores.

La necesidad de generación de oportunidades para todos.

El ritmo acelerado de los procesos de integración regional-mundial.

El crecimiento progresivo del desarrollo de los sistemas de C-I-T.

Estas dinámicas sociales han determinado un fuerte crecimiento y diversificación de los sistemas educativos, haciendo que en ellos se de, en tiempos muy cortos, un gran incremento de la matricula de estudiantes nuevos y del profesorado, observándose en éste cantidades deficientes y niveles de formación no acordes con las demandas que recibe el sector: se presenta además el desconocimiento de la calidad del sistema, de las instituciones que lo conforman y de los programas que éstas ofrecen.

Se reta pues a la universidad para que desarrolle o afine sus capacidades para responder por:

Una mayor cobertura (más cupos, nuevas sedes,nuevas íes, más regionalización)

Una mayor calidad.

Una mayor equidad.

Una mayor pertinencia

Una mayor formación de recurso humano altamente especializado y

Contribuir más significativamente al desarrollo de la C-I-T, 
Lo que implica:

Más investigación, más programas de maestría y de doctorado.

En este ámbito de transformaciones y demandas de nuevos roles de la educación superior se presenta una recomposición de las relaciones de la universidad con el Estado y con los sectores productivos, se ajusta cada una de las agendas y se definen campos de convergencia que aproximan los intereses de la educación superior con los intereses generales de la sociedad.

Esta recomposición de relaciones tiene como substrato algunos postulados básicos, tales como: la valorización del conocimiento y con ella la identificación de la educación como factor de equidad y uno de los motores del desarrollo científico, social, económico y cultural. Consustancial a la anterior, el reconocimiento de que la inversión en educación de calidad es condición para el logro del desarrollo.

Se hace visible el papel clave que entra a jugar la tríada cobertura-calidad-financiación. Pero paradójicamente se presenta la crisis de financiación de la educación por el Estado y se buscan nuevas estrategias; la financiación de la demanda, vía crédito educativo, los recursos propios, vía venta de servicios, los donativos, fondos concursables.

Un punto claro de convergencia lo constituye la necesidad del aseguramiento de la calidad. Un sistema educativo diferenciado por sus niveles de calidad hace que la equidad que se pretende lograr con los incrementos de cobertura pierda eficacia y que la inversión que en ella se hace por parte de las familias, del Estado y de sectores externos pierda eficiencia, lleva a que la sociedad pierda la oportunidad de tener conocimientos de alta pertinencia y recursos humanos con los niveles de cualificación exigidos por la sociedad del conocimiento.

A lo largo del texto ronda la conexión evaluación universitaria-calidad de la educación/calidad de la universidad y es que evaluar la universidad bajo la orientación de una real voluntad del mejoramiento o transformación de las estructuras y prácticas académicas y de gestión implica evaluar la calidad del sistema, de sus instituciones y de sus ofertas educativas, como estrategia de la política educativa Estatal e Institucional.

\section{La calidad de la educación superior.}

Al referirse a la calidad se hace referencia a:

Aquello que reviste cierta excelencia socialmente reconocida 
Aquello que llena nos estándares mínimos previamente establecidos.

Situaciones en las que las cosas se hacen con cero defectos.

La capacidad de alcanzar objetivos propuestos.

Capacidad de generar conocimiento estructural en la organización.

Proceso omnidireccional cuyos resultados difieren dependiendo de los patrones de Referencia.

En el entendimiento de la necesidad de una definición clara de la calidad para poder lograr su evaluación, el CNA de Colombia ha adoptado las siguientes definiciones: "Aquello que determina la naturaleza de algo, como aquello que hace de algo lo que ese algo es".

"La calidad de algo es la medida en que ese algo se aproxima al prototipo ideal, definido históricamente como realización optima de lo que le es propio según el género al que pertenece".

Las dos anteriores definiciones se refieren a la posibilidad de:-distinguir algo como perteneciente a un género y -distinguir entre los diferentes miembros de un género y entre ellos y el prototipo ideal definido para ese género.

Al aproximar estos conceptos de calidad a la educación superior el CNA propone:" Hace referencia a la síntesis de características que permiten reconocer un programa académico específico o una institución de determinado tipo, y hacer un juicio sobre la distancia relativa entre el modo como en esa institución o programa se presta ese servicio y el óptimo que corresponde a su naturaleza.

Como proceso académico que es la evaluación de la calidad de la educación, de sus instituciones y programas, es la universidad, como nicho productor y reproductor de los campos de saber y de las comunidades académicas, la institución idónea para definir su calidad y diseñar los instrumentos y mecanismos para su evaluación y mejoramiento.

La evaluación y el aseguramiento de la calidad se toman en dispositivos prioritarios para la orientación de políticas de:

Desarrollo estratégico de la institución.

Para la orientación de la institución por una cultura del aseguramiento de la calidad y de la autorregulación.

Para la orientación de procesos de descentralización.

Para el fomento y apoyo a las prácticas de rendición de cuentas (accountability). 
Para el fomento del ejercicio responsable de la autonomía.

Para la regulación de relaciones inter e intrainstitucionales.

Para la identificación de fortalezas, debilidades y oportunidades.

Para la incorporación de la institución en los sistemas de acreditación nacional e internacional. En síntesis para apalancar el mejoramiento continuo e integral de la institución y de sus programas.

\section{Acreditación y Evaluación:}

Como ya se ha mencionado la acreditación y la evaluación son dos dispositivos estratégicos que permiten orientar los desarrollos de la institución y de los programas. Ellas no son fines en sí, son medios, son instrumentos que la institución acoge y los utiliza según sus intereses y condiciones específicas.

Mediante la acreditación las agencias u organismos de acreditación dan FE PÚBLICA de la calidad que posee un programa, alcanzada por el logro de sus postulados misionales, por el cumplimiento de sus objetivos, por la pertinencia de su misión y proyectos.

En los distintos sistemas de acreditación se incorpora la evaluación como su componente fundamental y se hace bajo dos formas: la auto evaluación y la heteroevaluación o evaluación externa o más conocida como la evaluación por pares externos.

La autoevaluación es el ejercicio interno, propio de la institución o del programa, para la realización del examen, de la propia lectura por parte de los académicos y demás actores que participan del diseño y ejecución de las misiones, funciones, objetivos, proyectos y acciones mediante las cuales se realizan la institución y sus diversos programas.

Este ejercicio reclama la participación de los profesores- académicos-, directivos-académicos y técnicos-, estudiantes, egresados y aquellos focos poblacionales hacia quienes se dirigen sus productos. Es este elemento el que le da el carácter de "proceso participativo". Reclama de sus agentes plena objetividad y transparencia

La evaluación externa es la lectura que la comunidad académica y científica, externa a la institución y al programa hace de ellos. Es una evaluación sistemática, integral realizada en condiciones normales de funcionamiento de la institución o del programa y es mediada por los informes generados en la autoevaluación. 
Esta evaluación parte del reconocimiento de las comunidades académicas y científicas y reclama de quien funja como evaluador que sea, en el sentido Kuhntiano, un par, que sea "primus interpares",identificado profesionalmente como alguien que posee la autoridad que le permite emitir un juicio sobre la calidad de un programa o institución; interlocutor válido frente a la comunidad del programa o institución.

Entendidos así los ejercicios de auto evaluación y evaluación externa y las relaciones que entre ellas se establecen se hace claro el principio de que "la evaluación es el ejercicio de la academia para examinar la academia" y que al Estado y a los entes externos sólo les es dado armarse de los criterios y mecanismos que aquella les proporciona.

Dentro de este marco de comprensión de la evaluación universitaria es válida la pregunta por los retos que ella plantea a la Universidad y demás Instituciones de Educación superior y a Los organismos de acreditación.

\section{Debe reconocerse en primer lugar que:}

La educación no es responsabilidad exclusiva del Estado, de la sociedad o de la institución educativa. La educación es una responsabilidad de todos, es una responsabilidad compartida.

Dentro de la institución tampoco es responsabilidad exclusiva de alguno de sus actores; directivos, profesores, estudiantes, personal técnico-administrativo y demás actores institucionales son responsables por el servicio educativo que la institución presta.

La educación es un servicio público en el cual está comprometido el interés general por contar con instituciones que acrecienten el conocimiento en todos los campos, formen profesionales y preparen el talento para el desarrollo científico y tecnológico.

La universidad es un ente académico dotado de autonomía académica, financiera y administrativa. Es un imperativo ético institucional darle a ese servicio la mayor calidad.

\section{Corresponde a los Organismos de acreditación:}

Reconocer plenamente la autonomía de la institución y preservarla durante el proceso de 
acreditación.

Reconocer a las comunidades académicas y ser interlocutor válido de ellas para el establecimiento de criterios y procedimientos.

Establecer reglas de juego claras y visibles que orienten los procesos de acreditación de instituciones y programas.

"Por medio de la acreditación, ser garante de la calidad de los programas e instituciones. Participar, con el Estado y las instituciones, del desarrollo de las políticas de la educación superior y ser ente activo del mejoramiento de la calidad de la educación superior.

Diseñar criterios y mecanismos para la selección e inducción del par como evaluador externo. Vigilar la validez de los instrumentos incorporados en esta labor y la calidad de los informes que en ella se produzcan.

Comprometerse con el aseguramiento de su propia calidad y en consecuencia realizar su autoevaluación y someterse a la evaluación por pares.

Contribuir al diseño de criterios y mecanismos, para el aseguramiento de la calidad de las agencias, bajo parámetros reconocidos por diversos gobiernos y órganos de acreditación que faciliten ambientes de confianza y consensos básicos, sobre los cuales se sustenten el mutuo reconocimiento de los organismos acreditado res y procesos de cooperación interregional.

Participar en iniciativas de armonización de los criterios y procedimientos de evaluación y acreditación de instituciones y programas de pregrado, maestrías y doctorados que faciliten procesos de cooperación interinstitucional.

Ser parte constitutiva de redes de conocimiento del campo educativo y en particular del área de la calidad, la evaluación y la acreditación de la educación superior.

\section{Corresponde a la Institución Universitaria:}

Reconocer el mejoramiento de la calidad de la educación como una exigencia de la sociedad y a la evaluación como un dispositivo idóneo para la obtención de la información requerida para la regulación de la institución y de sus programas de formación.

Incorporar y fomentar la evaluación como un elemento de la cultura institucional y como tal irrigarla por todas sus dependencias académicas y administrativas, por sus actividades y programas.

Hacer de la evaluación una política institucional con la cual se comprometan los diferentes órganos de dirección de la institución-C superior, C académico, C de facultad, C de programa- y los entes académico-administrativo-V Rectorías y direcciones-. 
Dotar a la institución de las estructuras administrativas y de los recursos adecuados para el desarrollo de la evaluación y del aseguramiento de la calidad.

Formar la "masa crítica" institucional capaz de liderar y conducir los procesos de evaluación y aseguramiento de la calidad.

Estimular la participación de los diferentes actores de la comunidad universitaria en los procesos de evaluación y en el diseño, ejecución y seguimiento de las acciones de mejoramiento resultantes de ellos.

Adoptar modelos sistémicos de evaluación que se correspondan con definiciones comprensivas de la calidad, de forma tal que al evaluarse o al evaluar sus programas incluya los insumos, los procesos, los productos y el contexto y logre comprender la calidad como determinada por cada uno de ellos y por sus interrelaciones.

Hacer uso coherente de los resultados de la evaluación en los procesos de planeamiento estratégico institucional y del mejoramiento continuo de la calidad y pertinencia de la institución y de los programas.

Conducir sus procesos de evaluación con criterios de reconocimiento internacional para potenciar la internacionalización de las institución y favorecer la comparabilidad y_...

Homologación de los estudios cursados en ella y de los títulos por ella otorgados y la movilidad de sus programas, estudiantes y profesores.

Participar en procesos de armonización de criterios y mecanismos de evaluación y aseguramiento de la calidad y adoptar sus resultados, con el fin de dotar a la institución y a los programas de características que los pongan a punto para formar parte competente de los espacios y programas de conocimiento y de cooperación regional e interregional'

Comprenderse como punto de redes amplias de conocimiento y participar mediante la evaluación de los sistemas de investigación, maestría y doctorado en la consolidación del modo 2 de producción del conocimiento. 
Incorporarse en los Sistemas de Acreditación y obtener la fe pública de la calidad de sus programas e incrementar su legitimidad como institución de saber al servicio de la sociedad.

Participar con los órganos Estatales (MEN) y las agencias de acreditación en la construcción de consensos sólidos sobre las políticas, criterios y mecanismos para el fomento de la calidad del sistema educativo, de sus instituciones y programas.

Apoyarse en los procesos de evaluación para "la rendición de cuentas" a la comunidad universitaria, al Estado ya la sociedad.

Hacer de la evaluación un instrumento para la conducción de la institución por la cultura y las prácticas de la autorregulación y realizar de manera conciente e informada, por el conocimiento permanente y detallado de sus diversos factores, el postulado de que "la transformación de la universidad sólo puede ser realizada por la universidad misma, con un sólido compromisoparticipación- de la comunidad que la sostiene". 